\title{
Development and effects of a psychosocial adaptation program for North Korean adolescent defectors
}

\author{
In-Sook Lee ${ }^{1}$, Jung-Hee Jeon ${ }^{2}$ \\ ${ }^{1}$ Associate Professor, Department of Nursing, Hannam University, Daejon; ${ }^{2}$ Visiting Professor, College of Nursing, Ewha Womans University, Seoul, Korea
}

Purpose: This study aimed to develop and examine the effects of a psychosocial adaptation program for North Korean adolescent defectors. Methods: This quasi-experimental study was conducted with 28 North Korean adolescent defectors who were recruited through alternative schools. The experimental and control groups consisted of 14 participants each. A program was conducted across eight sessions, each lasting about 90-120 minutes. The data were analyzed using the $x^{2}$ test, $t$-test, paired t-test, and analysis of covariance. Results: No significant differences were observed in the score changes for psychosocial adaptation $(\mathrm{t}=0.25, p=.808)$, post-traumatic stress symptoms $(\mathrm{t}=0.32, p=.749)$, cultural adaptation stress ( $\mathrm{t}=0.05, p=.957)$, and self-efficacy $(\mathrm{t}=0.35, p=.726)$ in either group before and after the intervention. No intergroup differences were observed for psychosocial adaptation $(\mathrm{t}=0.12, p=.902)$, post-traumatic stress symptoms $(\mathrm{t}=0.07, p=.946)$, cultural adaptation stress $(\mathrm{t}=0.01, p=.994)$, and self-efficacy $(\mathrm{t}=0.58, p=.570)$. Conclusion: These findings suggest that psychosocial adaptation, stress, and self-efficacy cannot be improved by a short-term intervention program alone. It was also determined that the cultural characteristics of the North Korean adolescent defectors were not sufficiently considered before implementing the program. Therefore, it is essential to provide an in-depth orientation for the participants before implementing the program.

Key words: Democratic People's Republic of Korea; Adolescent; Adaptation, psychological; Program

\author{
Corresponding author \\ In-Sook Lee \\ Department of Nursing, Hannam \\ University, 70 Hannam-ro, Daedoek-gu, \\ Daejon 34430, Korea \\ TEL: +82-42-629-8472 \\ FAX: $+82-42-629-8472$ \\ E-MAIL: is2009@hnu.kr
}

This study was supported by a National Research Foundation of Korea(NRF) grant funded by the Korean government(No. NRF-2016R1D1A2B03930448).

Received May 11, 2021

Revised Jun 6, 2021

Accepted Jun 27, 2021

This is an Open Access article distributed under the terms of the Creative Commons Attribution NonCommercial License (http://creativecommons.org/licenses/by-nc/4.0/) which permits unrestricted noncommercial use, distribution, and reproduction in any medium, provided the original work is properly cited.

\section{INTRODUCTION}

As the number of North Korean defectors has steadily risen in recent years, the number of school-age adolescents among them has also increased, totaling 2,531 as of April 2019 [1]. North Korean adolescent defectors often experience traumatic events in the course of leaving North Korea and arriving in South Korea, including threats to their personal safety, parting with their families, and fear of forceful repatriation to North Korea, which can negatively impact their personal development and resettlement in South Korean society. Studies have shown that traumatic experiences during the sensitive adolescent period threaten normal development, putting adolescent defectors at risk of impaired physical maturation and health and predisposing them to anxiety, inferiority complexes, identity crises, and lasting depression, all of which ad- versely affect their adaptation to South Korean society [2]. North Korean adolescent defectors, who are in the process of forming a sense of identity and preparing for adulthood, have a high risk of developing psychosocial problems such as confusion, depression, and frustration resulting from conflicts between their identities formed within the North Korean education system and the ways of thinking required to be part of South Korean society [3].

Apart from negatively affecting individual North Korean adolescents who defect, these problems also lead to wider social problems such as discrimination against and marginalization of North Korean adolescent defectors. In order to break the cycle, there is an urgent need not only to resolve defectors' inner conflicts and minimize the problems they encounter in South Korean society, but also to nurture their ability to actively adapt to and solve problems in society [4]. 
Most North Korean adolescent defectors show symptoms of anxiety due to having experienced hunger, hardship, separation from their families in North Korea, extreme fears during the defection process and their time in China, concerns about the unfamiliar culture of South Korea, and anxiety and uncertainty about the future [3]. The double burden of social vulnerability and rapid physical and psychological changes during adolescence can further amplify various stresses North Korean adolescent defectors experience, including post-traumatic stress symptoms (PTSS), and their consequences. In addition, as they confront a cultural landscape in South Korea that is completely different from the North Korean culture they came from, North Korean adolescent defectors can experience alienation, confusion, and stress during cultural adaptation due to culture shock or prejudice and discrimination from their peers [5]. In particular, the social discrimination to which they are exposed during the sensitive period of puberty can lead to a decrease in self-efficacy and self-confidence, which in turn weakens social adaptation and achievement, reinforcing an endless cycle of discrimination. Discrimination has been shown to be significantly correlated with psychological stress and negatively correlated with self-esteem and self-efficacy [6]. Thus, enhancing the self-efficacy of adolescent defectors is especially important for disrupting the vicious cycle. Self-efficacy is the conviction that one can successfully perform an action to produce the desired outcome-that is, one's own judgment of his or her ability to execute a chosen action. Self-efficacy, in other words, influences the thought processes and emotional responses that determine one's chosen actions and the intensity and extent of one's efforts to execute those actions [7]. Allen et al. [8] found that self-efficacy was the driving force behind persevering at a task by motivating people to adjust to their environment, have self-determination, and act with creativity and enthusiasm even in environments with many risk factors. Therefore, self-efficacy must be fostered to develop social adaptation abilities and acquire cognitive skills. Moreover, North Korean adolescent defectors face various struggles depending on their personal backgrounds, their reasons for defection, the time during which they stayed in another country before South Korea and what they experienced there, and family status before entering South Korea. After their entry into South Korea, adolescent defectors encounter problems associated with abrupt familial and sociocultural changes coupled with difficulties adapting to new schools and sociocultural settings due to rampant prejudice in South Korean society and a sense of alienation from South Korean culture and society [9]. Given the current lack of efficient adaptation intervention measures, support plans must be developed to raise public awareness and support for North Korean adolescent defectors and facilitate their socio- cultural adaptation.

Socioeconomic minority groups and ethnic minorities reportedly have an elevated risk of emotional disorders, with the rate of untreated serious affective problems among children ranging from $3 \%$ to $26 \%$, which highlights the need to design and implement effective intervention programs to improve their social adaptation, response skills, and emotional health [10]. A previous study found that such intervention programs reinforce positive emotional and social adaptation skills, control anxiety and depression, improve adverse school situations, and prevent substance abuse problems that persist into adulthood. It has also been found that important factors affecting the adaptation of children and the children themselves should be identified and addressed in intervention programs accordingly [10]. With respect to social and cultural factors that affect adaptation, North Korean adolescent defectors should be provided with support to improve sociocultural adaptation, psycho-emotional adaptation, and school-related adaptation, as well as social support in the form of individual or family-centered counseling and family and peer support aimed at refugee children and adolescents [11].

Research on the psychosocial characteristics and adaptation status of North Korean adolescent defectors, however, remains at a basic level. Programs on building career experience and employment support were developed in the early stages with no procedures to verify their effectiveness [12]. There is a lack of research studying the application of integrative interventions to reduce stress, improve self-efficacy, and promote psychosocial adaptation by strengthening social support. To our knowledge, there have been no studies in which integrated psychosocial adaptation promotion programs were implemented.

This study, therefore, aimed to lay the foundation for the development of a psychosocial adaptation program suitable for North Korean adolescent defectors by devising and implementing a preliminary program to improve their psychosocial adaptation and evaluating its effectiveness.

\section{Research Purpose}

This study aimed to develop a program to facilitate North Korean adolescent defectors' psychosocial adaptation to South Korean society and evaluate its effectiveness. To this end, two concrete research objectives were formulated.

- An integrative creative-cognitive-cooperative-adaptation promotion (3CAP) program for North Korean adolescent defectors was developed.

- The 3CAP intervention program was implemented and its effects were evaluated. 


\section{METHODS}

Ethics statement: This study was approved by the institutional review board of Hannam University (No. 2018-03-01-1113). Informed consent was obtained from the participants.

\section{Research Design}

This was a quasi-experimental study that used a non-equivalent control group pretest-posttest design aimed at developing a psychosocial adaptation program and evaluating its effects on self-efficacy, stress, and psychosocial adaptation in North Korean adolescent defectors (Figure 1).

\section{Participants}

Twenty-eight students were recruited from among North Korean adolescent defectors who attended an alternative school and a government-funded middle and high school for North Korean refugees adjusting to life in South Korea. The inclusion criteria were as follows: adolescents (12-20 years old) without signs of developmental delays; adolescents with adult kin or a non-kin caregiver (e.g., legal guardian), both of whom understood the purpose and procedures of the study and provided written informed consent; and adolescents who could communicate well enough to understand and answer questions. The criteria for exclusion of subjects who may not have been able to proceed with the study or who may have affected the results of the study due to participation in other programs were as follows: adolescents who were undergoing hospital treatment for diseases or who had been diagnosed with an infectious disease or any disease requiring hospitalization; adolescents with physical disabilities or cognitive and affective disorders; and adolescents with prior experiences participating in a similar program.

The sample size was determined using the $G^{*}$ Power 3.0 program by applying the difference between two independent means (two groups) with a significance level of .05, power of $80 \%$, and effect size of .80 [13]. Of the 42 adolescents who met the above selection criteria, 21 subjects were assigned to the experimental group and 21 to the control group. First, to prevent the diffusion of the experimental treatment, 21 subjects were assigned to the control group and their data were collected, which was followed by implementation of the program for the 21 additional subjects in the experimental group. After excluding subjects who had missing or incomplete data, lacked understanding of the intervention process, stopped attending the program due to their school schedules, withdrew their voluntary participation, or withdrew their questionnaire responses, data obtained from 28 subjects (14 from each group) were ultimately analyzed.

\section{Development of a 3CAP Program}

The purpose of the program in this study was to relieve stress such as psychological anxiety and isolation felt by North Korean adolescents, increase their self-efficacy, promote mutual understanding and nurturing relationships between North and South Korean adolescents, and develop a psychosocial adaptation program that can help North Korean adolescents integrate into the local community.

Three types of interventions are known to promote refugee children and adolescents' psychosocial adaptation: 1) creative expression, 2) cognitive-behavioral therapy, and 3) various strategic models. Academic performance and general well-being were found to improve as a result of such interventions [11].

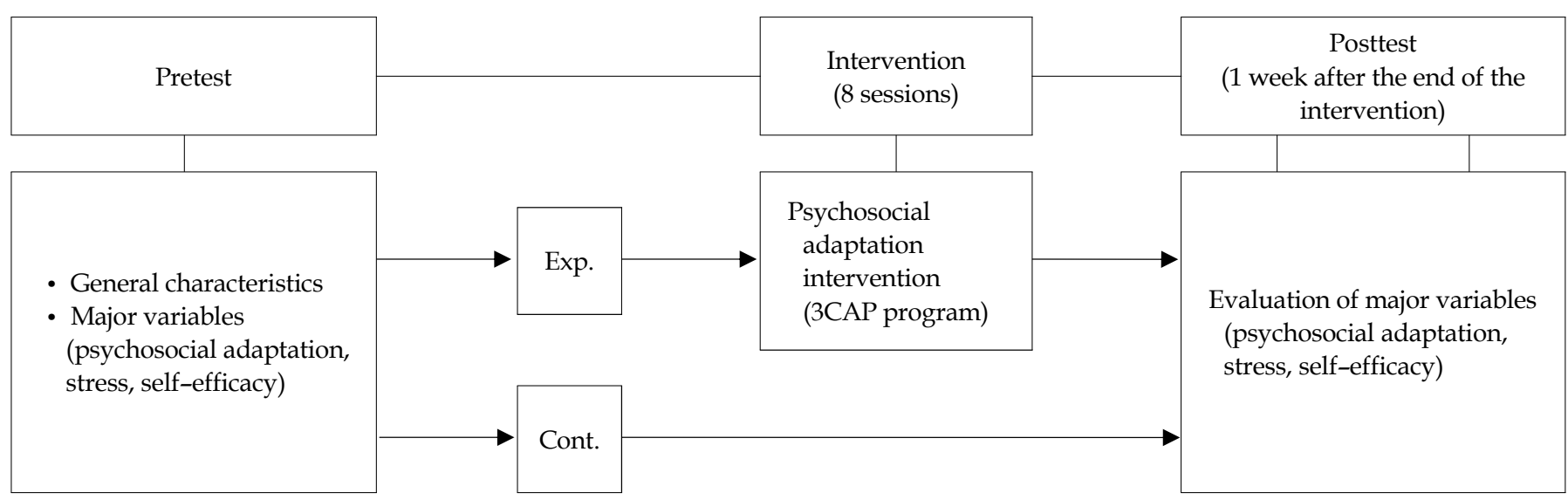

Figure 1. Research design. 3CAP, creative-cognitive-cooperative-adaptation promotion program; Cont., control group; Exp., experimental group. 
Creative expression intervention utilizes art (drawing and writing expression) and music to help people express their emotions through creative expression and play therapy. In particular, a shared musical culture is helpful for adaptation and cultural assimilation beyond ethnicity. Through dancing and singing, self-confidence and peer relationships have been found to improve [14].

Cognitive-behavioral intervention helps reduce post-traumatic stress disorder (PTSD), depression, anxiety and emotional-behavioral problems in children who have experienced trauma and improves their stress management and coping skills. Similar to creative expression intervention, cognitivebehavioral intervention improves the communication and decision-making processes of subjects using positive rewards and empathy as well as replaces one's negative thoughts with positive and rational thoughts. Cognitive-behavioral intervention is thus expected to help North Korean adolescent defectors properly express their thoughts, feelings, and actions [15]. Therefore, this study aimed to reduce the stress experienced by North Korean adolescent defectors after entering South Korea and promote psychosocial adaptation through dialogue with their peers and group activities.

Functional social support that refugees and migrants need can be divided into three categories: (1) instrumental support that involves material aid, such as financial aid or direct help with daily tasks, (2) informational support that involves sharing relevant information in the form of advice or guidance on how to cope with their situation, and (3) emotional and psychological support using empathy, reassurance, caring, and trust [16]. Thus, the cooperative efforts between the different types of social support and mutual understanding play a crucial role in helping individuals adapt and recover in various ways [16]. In addition, North Korean adolescent defectors must overcome cultural pressures, such as feelings of cultural deficiency and inferiority, caused by their sudden exposure to popular culture and media, the culture of their South Korean peers, and appearance-related cultural pressures [5].

Therefore, in this study, a 3CAP program, referring to the three Cs (creative, cognitive, and cooperative), was developed. It was designed to facilitate psychosocial adaptation and the integration of North Korean adolescent defectors as members of the South Korean community. In particular, to encourage mutual cooperation, the program included activities in which participants would interact with a South Korean peer group for social support. The South Korean peer groups provided information on popular culture such as food, music, and dance to create opportunities for mutual understanding. In addition, the program provided the participants with information on trendy makeup methods and styles adopted by South Korean teenagers. By giving the participants the tools they needed to cultivate their image, the psychosocial adaptation program was designed to alleviate North Korean adolescent defectors' psychological anxiety and isolation, increase their self-efficacy, enhance their mutual understanding and connection with their South Korean peers, and integrate them as members of their local communities. Previous studies were reviewed to take the characteristics of North Korean adolescent defectors into account when designing the program, and the following factors affecting psychosocial adaptation were determined to be the outcome variables: self-efficacy, PTSS, culture adaptation stress, and psychosocial adaptation.

The outcome variables that comprised the main factors affecting psychosocial adaptation such as self-efficacy, PTSS, and acculturation stress $[3,5,9]$ were incorporated into the content and methods of the program based on previous studies $[3,10,11,13-15,17,18]$, and advice from related experts was sought to ensure the validity of the design and contents of the program and to increase its effectiveness. Two professors of nursing with specializations in children and adolescents with experience in North Korean defector research, one nurse with more than 20 years of experience at Hanawon (the settlement support center for North Korean refugees), and one pediatrician were consulted about the physical and mental condition of North Korean adolescent defectors. To gauge the North Korean adolescents' understanding of South Korea, the program was also individually evaluated by two teachers who worked at Hanawon and was revised and supplemented accordingly.

Based on advice from the experts regarding the recommended number of intervention sessions, the ideal session duration, and the recommended program contents to be included for participating students while also considering a feasible intervention and data collection period, a total of eight sessions were planned which would be conducted on a twice per week basis. The content of the 3CAP program is shown in Table 1.

\section{Experimental Treatment}

Each session was conducted by either the researchers (the first, second, seventh, and eighth sessions) or by the participants' South Korean peers (the third, fourth, fifth, and sixth sessions). The session duration ranged from 90 to 120 minutes per session. Sessions were conducted twice per week over a total of four weeks using the time (6-8 pm) after the subjects' regular classes in the Hanawon seminar room. The scope of each session was as follows: orientation (session 1), empathy (session 2), catching up with South Korean culture (sessions 3 and 4), self-expression through cooking classes (sessions 5 and 6), designing one's image (session 7), and preparing for 
Table 1. Organization and Content of the 3CAP Program

\begin{tabular}{|c|c|c|c|c|c|}
\hline \multicolumn{2}{|c|}{ Session } & \multirow{2}{*}{$\begin{array}{c}\begin{array}{c}\text { Length } \\
\text { (minutes) }\end{array} \\
90\end{array}$} & \multirow{2}{*}{$\begin{array}{l}\text { Method } \\
\begin{array}{l}\text { Lecture, } \\
\text { presentation }\end{array}\end{array}$} & \multirow{2}{*}{$\begin{array}{l}\text { Session contents } \\
\text { - Introducing the 3CAP program } \\
\text { - Getting to know one another and } \\
\text { interacting } \\
\text { - Understanding and accepting } \\
\text { differences } \\
\text { - Writing to express feelings and } \\
\text { experiences }\end{array}$} & \multirow{2}{*}{$\begin{array}{l}\text { Additional comments } \\
\text { - Introducing 3CAP } \\
\text { - Learning how to interact while introducing } \\
\text { oneself and others } \\
\text { - Writing about one's emotions related to the } \\
\text { experience of escaping from North Korea; } \\
\text { talking about one's experiences on the talk } \\
\text { ball }\end{array}$} \\
\hline 1 & $\begin{array}{l}\text { Orientation, } \\
\text { program } \\
\text { explanation }\end{array}$ & & & & \\
\hline 2 & Feeling empathy & 90 & $\begin{array}{l}\text { Lecture, } \\
\text { presentation }\end{array}$ & $\begin{array}{l}\text { - Describing one's own } \\
\text { experiences or the experiences of } \\
\text { their family members } \\
\text { - Art or drawing activity }\end{array}$ & $\begin{array}{l}\text { - Sharing feelings and experiences (talk ball } \\
\text { concert) } \\
\text { - Encouraging free expression } \\
\text { - Gauging negative feelings and thoughts } \\
\text { and stepping in to alleviate them }\end{array}$ \\
\hline 3 & $\begin{array}{l}\text { Knowing about } \\
\text { South Korean } \\
\text { culture }(\mathrm{I})\end{array}$ & 120 & $\begin{array}{l}\text { Lecture, } \\
\text { demonstration } \\
\text { practice }\end{array}$ & $\begin{array}{l}\text { - Watching a TV show or a DVD } \\
\text { and having a discussion, } \\
\text { comparing the video to what they } \\
\text { knew about South Korean culture }\end{array}$ & $\begin{array}{l}\text { [Catching up with idol singers] } \\
\text { - Sharing knowledge about South Korean } \\
\text { (youth) culture and comparing their } \\
\text { knowledge through discussion } \\
\text { - Group activity; shared interest }\end{array}$ \\
\hline 4 & $\begin{array}{l}\text { Knowing about } \\
\text { South Korean } \\
\text { culture (II): } \\
\text { Sharing emotions } \\
\text { in South Korea }\end{array}$ & 120 & $\begin{array}{l}\text { Lecture, } \\
\text { demonstration } \\
\text { practice }\end{array}$ & $\begin{array}{l}\text { - Sharing Korean pop } \\
\text { - Creating songs and lyrics in } \\
\text { order to create a "happy } \\
\text { adaptation" }\end{array}$ & $\begin{array}{l}\text { [Learning Korean pop rap from an aspiring } \\
\text { rapper] } \\
\text { - Helping to better adapt to South Korean } \\
\text { culture through singing and dancing using } \\
\text { Korean pop as a culture without ethnic } \\
\text { barriers } \\
\text { - Controlling emotions and behaviors } \\
\text { through songwriting and composition }\end{array}$ \\
\hline 5 & $\begin{array}{l}\text { Developing } \\
\text { communication } \\
\text { skills via a cooking } \\
\text { class with a future } \\
\text { chef }\end{array}$ & 120 & $\begin{array}{l}\text { Lecture, } \\
\text { demonstration } \\
\text { practice }\end{array}$ & $\begin{array}{l}\text { - Understanding how thoughts } \\
\text { and feelings affect behavior } \\
\text { - Role-play feeling happy, sad, } \\
\text { scared, and angry } \\
\text { - Identifying barriers to effective } \\
\text { communication } \\
\text { - Learning effective communication } \\
\text { skills } \\
\text { - Cooperate and communicate } \\
\text { effectively with others }\end{array}$ & $\begin{array}{l}\text { [Making snacks with a future chef I] } \\
\text { - Improving communication skills while } \\
\text { cooking with a friend who is an aspiring } \\
\text { chef } \\
\text { - Helping children and adolescents who face } \\
\text { difficulties building peer relationships due } \\
\text { to language barriers by teaching them } \\
\text { communication skills using effective } \\
\text { methods }\end{array}$ \\
\hline 6 & $\begin{array}{l}\text { Learning coping } \\
\text { strategies via a } \\
\text { cooking class with } \\
\text { future chef }\end{array}$ & 120 & $\begin{array}{l}\text { Lecture, } \\
\text { demonstration } \\
\text { practice }\end{array}$ & $\begin{array}{l}\text { - Discussions about difficulties } \\
\text { associated with having defected } \\
\text { from North Korea and coping } \\
\text { strategies related to adapting to } \\
\text { South Korea } \\
\text { - Individual and group } \\
\text { problem-solving skills } \\
\text { - Building leadership skills, making } \\
\text { a decision, resolving conflict, etc. }\end{array}$ & $\begin{array}{l}\text { [Making snacks with a future chef II] } \\
\text { - Using brainstorming or role-playing to } \\
\text { improve decision-making and } \\
\text { problem-solving abilities and improve } \\
\text { interpersonal relationships and stress } \\
\text { responses } \\
\text { - Acquiring communication and leadership } \\
\text { skills while learning how to cook }\end{array}$ \\
\hline 7 & $\begin{array}{l}\text { [Self-esteem } \\
\text { development] } \\
\text { Self-awareness \& } \\
\text { establishing role } \\
\text { modeling }\end{array}$ & 120 & $\begin{array}{l}\text { Lecture, } \\
\text { demonstration } \\
\text { practice }\end{array}$ & $\begin{array}{l}\text { - Cultivating one's image } \\
\text { - Improving self-esteem } \\
\text { - Developing self-discipline, } \\
\text { resilience, adaptability, and } \\
\text { self-management } \\
\text { - Role modeling }\end{array}$ & $\begin{array}{l}\text { [Makeup that makes me shine] } \\
\text { - Enhancing self-esteem necessary for } \\
\text { adaptation, building self-awareness, } \\
\text { learning self-management skills, and } \\
\text { finding a role model }\end{array}$ \\
\hline 8 & $\begin{array}{l}\text { Preparing for the } \\
\text { future challenges }\end{array}$ & 90 & $\begin{array}{l}\text { Lecture, } \\
\text { presentation }\end{array}$ & $\begin{array}{l}\text { - Being proud and feeling rewarded } \\
\text { - Practicing praise } \\
\text { - Talking about opportunities and } \\
\text { discussing how to overcome } \\
\text { challenging situations }\end{array}$ & $\begin{array}{l}\text { - Wrapping up the program and learning } \\
\text { how to praise and reward oneself and } \\
\text { others } \\
\text { - Talking about future plans }\end{array}$ \\
\hline
\end{tabular}

3CAP, creative-cognitive-cooperative-adaptation promotion; DVD, digital video disc; TV, television. 
the future (session 8). It was explained to the students that if they failed to attend more than two sessions, according to the academic schedule of the school, they would be automatically excluded from the study.

The use of foreign languages and expressions in English was limited so that the North Korean adolescent defectors could fully understand the content of the program, and the program host and assistant participated in training and rehearsals for the intervention program.

\section{Instruments}

\section{1) Psychosocial adaptation}

The Korean Youth Self Report (K-YSR) [19] was used to assess the psychosocial adaptation of North Korean adolescent defectors. The K-YSR is the Korean version of the Youth Self Report [20], which was translated and standardized by Oh et al. [19]. For this study, the specific items from the K-YSR concerning withdrawal, depression/anxiety, delinquency, and aggression were used. The psychological adaptation subscale consists of 7 items concerning withdrawal and 16 items concerning depression/anxiety. A high score indicates a high degree of withdrawal and depression/anxiety (1=not true, 3= very often true). The social adaptation subscale consists of 11 items concerning delinquency and 19 items concerning aggression, with higher scores indicating a higher degree of maladjustment ( $1=$ not true, $3=$ very often true). Cronbach's $\alpha$ coefficient for the K-YSR [19] ranged from .63 to .85 at the time of its development. In this study, the total psychosocial adaptation score was obtained by adding the psychological and social adaptation scores. Cronbach's $\alpha$ coefficient for psychosocial adaptation in this study was .95 .

\section{2) Stress}

This study assessed two types of stress experienced by North Korean adolescent defectors: PTSS and cultural adaptation stress. Two psychometric tools were used after obtaining permission from their developers prior to data collection. PTSS were measured using a scale that was validated for use with North Korean adolescent defectors by Kim et al. [21]. The scale consists of 23 items in total, with 5 items on re-experiencing of the traumatic event, 7 items on avoidance, 5 items on hyperarousal, and 6 items assessing complex PTSD. Each item is rated on a 4 -point Likert scale $(0=$ not at all, $3=$ nearly every day). A higher score indicates more PTSS. Cronbach's $\alpha$ coefficient was .94 in the study by Kim et al. [21] and .96 in this study. Cultural adaptation stress was measured using a scale that was also validated for use with North Korean adolescent defectors by Kim et al. [21]. This scale consists of 5 subscales (discrimination, alienation, homesickness, cultural shock, and marginalization) covering a total of 16 items. Each item is rated on a 5 -point Likert scale ( $0=$ not at all, $4=$ very much). A higher score indicates a higher degree of cultural adaptation stress. Cronbach's $\alpha$ coefficient was .85 in the study by Kim et al. [21] and .87 in this study.

\section{3) Self-efficacy}

Self-efficacy, as used in a study of North Korean adolescents by Chung [22], was assessed based on the general self-efficacy scale developed by Cha [23]. It consists of 23 items, with 7 items on self-confidence, 12 items on self-regulatory efficacy, and 4 items on task difficulty preference, which were rated on a 5-point Likert scale ( 1 =strongly disagree, $5=$ strongly agree) with total possible scores ranging from 23 to 115 points. Higher scores indicate better self-efficacy. The original Cronbach's $\alpha$ coefficient for each subscale was .84 (self-confidence), .85 (self-regulatory efficacy), and .81 (task difficulty) [23]. In this study, the overall Cronbach's $\alpha$ coefficient for the scale was .76, and those for the three subscales were .69, .74, and .64, respectively.

\section{4) General characteristics}

The demographic characteristics of the subjects that were collected were sex, age, age at defection and entry, and education level before and after defection. Peer satisfaction, academic stress, and future anxiety were assessed using a Likert scale from 0 to 10 points. Higher scores in each corresponding category indicated greater peer satisfaction, greater academic stress, and greater anxiety about the future.

\section{Data Collection Methods and Procedure}

The research process carried out from November 2018 to February 2019 was as follows.

After obtaining permission from the heads of the two participating schools, we posted a recruitment advertisement on a bulletin board with the aid of homeroom teachers. The purpose and details of the program were explained to the students, who then voluntarily expressed their intention to participate in the study along with their caregivers (including non-family legal guardians). Those who gave written informed consent were enrolled in the study. The assistant researcher, assisted by the homeroom teacher when possible, then conducted a preliminary survey (pretest) on the participants' general characteristics and major variables (psychosocial adaptation, stress, and self-efficacy) prior to conducting the intervention program. Upon completion of the preliminary survey, the experimental group attended eight sessions of the program, which were administered twice a week for 1 month, to ensure the stability of the group. The educational ef- 
fect of the intervention program was assessed 1 week after its completion (posttest). The control group was issued the same pretest and posttest at the same time as the experimental group. The research outcomes were evaluated, and intergroup comparisons were drawn. Both groups were offered gifts as tokens of gratitude for participation.

\section{Ethical Considerations}

This study was conducted after obtaining approval from the institutional review board of Hannam University (approval number: 2018-03-01-1113). Approval for recruitment was obtained from the principals and homeroom teachers of the two participating schools.

\section{Data Analysis}

The collected data were analyzed using SPSS/WIN 21.0 (IBM Corp., Armonk, NY, USA). First, the $x^{2}$ test and t-test were performed to assess intergroup homogeneity and, given the sample size $(n=28)$, the Kolmogorov-Smirnov test was performed to verify the normality of the data on the depend- ent variables. The results showed that all of the quantitative variables had a normal distribution.

Second, the paired t-test was conducted to analyze the statistical differences in psychosocial adaptation, PTSS, cultural adaptation stress, and self-efficacy between the experimental group and the control group. Third, analysis of covariance was performed to confirm the differences (posttest scores) between the groups, with the pretest scores as a covariate.

\section{RESULTS}

\section{General Characteristics of the Participants}

No significant differences in the participants' general characteristics were observed between the experimental and control groups as a result of homogeneity testing (Table 2).

\section{Test of Baseline Homogeneity of Dependent Variables between Groups}

No significant differences were observed in the baseline scores for psychosocial adaptation, PTSS, cultural adaptation

Table 2. General Characteristics of the Participants $(N=28)$

\begin{tabular}{|c|c|c|c|c|c|}
\hline \multirow{2}{*}{ Characteristics } & \multirow{2}{*}{ Categories } & \multirow{2}{*}{$\begin{array}{c}\text { Exp. }(\mathrm{n}=14) \\
\mathrm{n}(\%) \text { or } \mathrm{M} \pm \mathrm{SD}\end{array}$} & \multirow{2}{*}{$\begin{array}{c}\text { Cont. }(\mathrm{n}=14) \\
\mathrm{n}(\%) \text { or } \mathrm{M} \pm \mathrm{SD}\end{array}$} & \multirow{2}{*}{$x^{2}$ or $\mathrm{t}$} & \multirow{2}{*}{$p^{*}$} \\
\hline & & & & & \\
\hline \multirow[t]{2}{*}{ Sex } & Male & $6(42.9)$ & $3(21.4)$ & \multirow[t]{2}{*}{ - } & \multirow[t]{2}{*}{.420} \\
\hline & Female & $8(57.1)$ & $11(78.6)$ & & \\
\hline Age (year) & & $17.1 \pm 2.1$ & $18.5 \pm 3.7$ & 1.19 & .243 \\
\hline Age of defection from North Korea (year) & & $16.6 \pm 2.2$ & $17.6 \pm 3.6$ & 0.95 & .352 \\
\hline Age of entry to South Korea (year) & & $16.9 \pm 2.2$ & $18.1 \pm 3.5$ & 1.03 & .312 \\
\hline \multirow[t]{3}{*}{ Education level (North Korea) } & People's school $^{\dagger}$ & $4(28.6)$ & $5(35.7)$ & \multirow[t]{3}{*}{-} & \multirow[t]{3}{*}{.379} \\
\hline & Junior high $^{\S}$ & $10(71.4)$ & $7(50.0)$ & & \\
\hline & $\geq$ College & $0(0.0)$ & $2(14.3)$ & & \\
\hline \multirow[t]{6}{*}{ Current education status } & Middle school & $2(14.3)$ & $4(28.6)$ & \multirow[t]{6}{*}{-} & \multirow[t]{6}{*}{.710} \\
\hline & High school & $4(28.6)$ & $1(7.1)$ & & \\
\hline & College & $2(14.3)$ & $2(14.3)$ & & \\
\hline & Alternative school & $5(35.7)$ & $5(35.8)$ & & \\
\hline & Qualifying exams & $1(7.1)$ & $1(7.1)$ & & \\
\hline & Employment preparation & $0(0.0)$ & $1(7.1)$ & & \\
\hline Peer satisfaction & & $8.3 \pm 1.3$ & $8.9 \pm 1.4$ & 1.13 & .270 \\
\hline Academic stress & & $5.4 \pm 2.8$ & $6.1 \pm 3.4$ & 0.55 & .590 \\
\hline Future anxiety & & $6.6 \pm 3.0$ & $6.4 \pm 3.6$ & 0.17 & .864 \\
\hline Psychosocial adaptation & & $75.9 \pm 19.3$ & $77.4 \pm 12.5$ & 0.23 & .818 \\
\hline PTSS & & $12.3 \pm 13.5$ & $14.5 \pm 9.5$ & 0.50 & .620 \\
\hline Cultural adaptation stress & & $22.9 \pm 11.2$ & $22.6 \pm 5.8$ & 0.11 & .917 \\
\hline Self-efficacy & & $76.2 \pm 8.9$ & $77.9 \pm 11.1$ & 0.45 & .657 \\
\hline
\end{tabular}

${ }^{*}$ t-test or Fisher's exact test; ${ }^{\dagger}$ Elementary school; ${ }^{\circledR}$ Middle-high school; Cont., control group; Exp., experimental group; PTSS, Post-traumatic stress symptom. 
stress, and self-efficacy between the experimental and control groups (Table 2).

\section{Effects of the Program on the Psychosocial Adapta- tion, Stress, and Self-efficacy of the Participants}

In the experimental group, the mean score for psychosocial adaptation decreased from $75.9 \pm 19.3$ in the pretest (baseline) to $73.6 \pm 17.1$ in the posttest ( 1 week after the intervention), but the difference was not statistically significant $(t=1.11, p=.289)$. It decreased from $77.4 \pm 12.5$ to $73.5 \pm 9.7(\mathrm{t}=0.47, p=.647)$ in the control group. No significant differences were observed in the score changes for psychosocial adaptation between the two groups $(\mathrm{t}=0.25, p=.808)$.

The mean PTSS score increased from $12.3 \pm 13.5$ to $12.9 \pm$ $14.4(t=0.15, p=.881)$ in the experimental group and decreased from $14.5 \pm 9.5$ to $13.6 \pm 7.3(\mathrm{t}=0.37, p=.721)$ in the control group. The score changes for PTSS showed no significant differences between the two groups $(t=0.32, p=.749)$.

The mean score for cultural adaptation stress decreased in both groups, from $22.9 \pm 11.2$ to $20.4 \pm 10.3(\mathrm{t}=1.23, p=.239)$ in the experimental group and from $22.6 \pm 5.8$ to $20.1 \pm 5.9(\mathrm{t}=$ $1.56, p=.143$ ) in the control group, but the changes were not statistically significant. No significant differences were ob- served in the score changes for cultural adaptation stress between the experimental and control groups $(t=0.05, p=.957)$.

The mean score for self-efficacy decreased from $76.2 \pm 8.9$ to $75.9 \pm 6.7(\mathrm{t}=0.18, p=.864)$ in the experimental group and increased from $77.9 \pm 11.1$ to $78.6 \pm 12.1(\mathrm{t}=0.33, p=.748)$ in the control group. The score changes for self-efficacy were not significantly different between the two groups $(t=0.35, p=.726)$ (Table 3).

No intergroup differences were observed in psychosocial adaptation scores $(t=0.12, p=.902)$, PTSS scores $(t=0.07, p=.946)$, cultural adaptation stress scores $(\mathrm{t}=0.01, p=.994)$, and self-efficacy scores $(\mathrm{t}=0.58, p=.570)$ (Table 4$)$.

\section{DISCUSSION}

North Korean adolescent defectors carry the burden of having to adapt to life in South Korea during a time of significant personal growth and development. Efforts to help adolescents adjust to society are effective only when they are already healthy.

A psychosocial adaptation program for North Korean adolescent defectors was developed in this study to test its effectiveness as an intervention. By doing so, we aimed to establish a social support network comprised of adolescent peers to

Table 3. Comparison of Psychosocial Adaptation, Stress, and Self-efficacy between Participants from Both Groups Before and After the Program

\begin{tabular}{|c|c|c|c|c|c|c|c|c|c|c|}
\hline \multirow{2}{*}{ Variables } & \multicolumn{4}{|c|}{ Cont. } & \multicolumn{4}{|c|}{ Exp. } & \multicolumn{2}{|c|}{$\begin{array}{l}\text { Difference in } \\
\text { both groups* }\end{array}$} \\
\hline & $\begin{array}{l}\text { Pretest } \\
\mathrm{M} \pm \mathrm{SD}\end{array}$ & $\begin{array}{l}\text { Posttest } \\
\mathrm{M} \pm \mathrm{SD}\end{array}$ & $\mathrm{t}$ & $p$ & $\begin{array}{l}\text { Pretest } \\
\mathrm{M} \pm \mathrm{SD}\end{array}$ & $\begin{array}{l}\text { Posttest } \\
\mathrm{M} \pm \mathrm{SD}\end{array}$ & $\mathrm{t}$ & $p$ & $\mathrm{t}$ & $p$ \\
\hline Self-efficacy & $77.9 \pm 11.1$ & $78.6 \pm 12.1$ & 0.33 & .748 & $76.2 \pm 8.9$ & $75.9 \pm 6.7$ & 0.18 & .864 & 0.35 & .726 \\
\hline Self-confidence & $21.0 \pm 4.6$ & $22.7 \pm 4.6$ & 1.70 & .112 & $23.6 \pm 4.6$ & $23.6 \pm 5.2$ & 0.07 & .949 & & \\
\hline Self-regulatory efficacy & $45.0 \pm 6.3$ & $44.1 \pm 6.7$ & 0.64 & .534 & $40.9 \pm 5.7$ & $39.8 \pm 6.4$ & 0.79 & .446 & & \\
\hline Task difficulty preference & $11.9 \pm 3.6$ & $11.7 \pm 4.4$ & 0.30 & .768 & $11.8 \pm 3.1$ & $12.4 \pm 2.7$ & 0.74 & .472 & & \\
\hline Cultural adaptation stress & $22.6 \pm 5.8$ & $20.1 \pm 5.9$ & 1.56 & .143 & $22.9 \pm 11.2$ & $20.4 \pm 10.3$ & 1.23 & .239 & 0.05 & .957 \\
\hline Discrimination & $2.9 \pm 1.8$ & $1.4 \pm 1.7$ & 2.39 & .033 & $3.6 \pm 1.8$ & $3.4 \pm 2.1$ & 0.34 & .738 & & \\
\hline Alienation & $4.2 \pm 2.4$ & $3.1 \pm 2.2$ & 1.92 & .078 & $4.9 \pm 3.0$ & $4.3 \pm 3.1$ & 1.14 & .273 & & \\
\hline Homesickness & $8.0 \pm 1.8$ & $8.2 \pm 1.9$ & 0.38 & .711 & $6.1 \pm 3.9$ & $6.9 \pm 3.0$ & 1.41 & .181 & & \\
\hline Cultural shock & $4.3 \pm 2.5$ & $4.4 \pm 2.5$ & 0.27 & .789 & $4.8 \pm 3.3$ & $3.3 \pm 3.0$ & 2.12 & .054 & & \\
\hline Marginalization & $3.2 \pm 2.0$ & $3.0 \pm 1.9$ & 0.56 & .583 & $3.6 \pm 2.1$ & $2.5 \pm 2.2$ & 1.75 & .104 & & \\
\hline PTSS & $14.5 \pm 9.5$ & $13.6 \pm 7.3$ & 0.37 & .721 & $12.3 \pm 13.5$ & $12.9 \pm 14.4$ & 0.15 & .881 & 0.32 & .749 \\
\hline Re-experience & $3.9 \pm 2.4$ & $2.9 \pm 3.0$ & 1.15 & .271 & $2.7 \pm 2.7$ & $2.9 \pm 2.8$ & 0.32 & .755 & & \\
\hline Avoidance & $3.4 \pm 3.6$ & $3.9 \pm 3.1$ & 0.63 & .541 & $3.1 \pm 3.7$ & $3.4 \pm 4.3$ & 0.36 & .725 & & \\
\hline Hyperarousal & $4.1 \pm 3.3$ & $4.1 \pm 2.7$ & 0.07 & .945 & $3.2 \pm 4.5$ & $2.9 \pm 3.1$ & 0.23 & .820 & & \\
\hline Complex PTSD & $3.1 \pm 2.3$ & $2.8 \pm 2.7$ & 0.43 & .671 & $3.3 \pm 4.8$ & $3.6 \pm 4.8$ & 0.19 & .854 & & \\
\hline Psychosocial adaptation & $77.4 \pm 12.5$ & $73.5 \pm 9.7$ & 0.47 & .647 & $75.9 \pm 19.3$ & $73.6 \pm 17.1$ & 1.11 & .289 & 0.25 & .808 \\
\hline Withdrawal & $11.4 \pm 2.3$ & $11.1 \pm 2.8$ & 0.61 & .555 & $10.1 \pm 3.0$ & $10.8 \pm 3.0$ & 1.13 & .280 & & \\
\hline Depression/Anxiety & $25.2 \pm 4.1$ & $24.3 \pm 4.7$ & 0.65 & .527 & $24.6 \pm 7.1$ & $24.3 \pm 7.6$ & 0.13 & .899 & & \\
\hline Delinquency & $14.5 \pm 3.2$ & $12.9 \pm 1.3$ & 2.16 & .050 & $14.5 \pm 3.5$ & $14.1 \pm 2.7$ & 0.42 & .681 & & \\
\hline Aggression & $27.4 \pm 5.8$ & $26.6 \pm 4.8$ & 0.65 & .524 & $28.1 \pm 8.0$ & $26.0 \pm 6.0$ & 1.12 & .285 & & \\
\hline
\end{tabular}

*Pretest-posttest; Cont., control group; Exp., experimental group; PTSD, post-traumatic stress disorder; PTSS, post-traumatic stress symptoms. 
Table 4. ANCOVA of the Psychosocial Adaptation, Self-efficacy, and Stress of the Participants

\begin{tabular}{llcccc}
\hline Variables & Group & B \pm SE & $t^{*}$ & $p$ & LSMEANS (95\% CI) \\
\hline Self-efficacy & Cont. & (ref.) & 0.58 & .570 & $78.0(74.1-81.8)$ \\
& Exp. & $-1.52 \pm 2.64$ & & $76.5(72.6-80.3)$ \\
Cultural adaptation stress & Cont. & (ref.) & 0.01 & .994 & $20.3(16.8-23.7)$ \\
& Exp. & $-0.02 \pm 2.36$ & & $20.2(16.8-23.7)$ \\
PTSS & Cont. & (ref.) & 0.07 & .946 & $13.1(7.50-18.7)$ \\
& Exp. & $0.26 \pm 3.86$ & & $13.4(7.8-19.0)$ \\
Psychosocial adaptation & Cont. & (ref.) & 0.12 & .902 & $73.2(66.2-80.3)$ \\
& Exp. & $0.60 \pm 4.83$ & & $73.8(66.8-80.9)$ \\
\hline
\end{tabular}

*These are results of ANCOVA of the difference between the two groups using scores from before the intervention as a covariate; ANCOVA, analysis of covariance; CI, confidence interval; Cont., control group; Exp., experimental group; PTSS, post-traumatic stress symptoms; ref., reference.

provide North and South Korean adolescents with opportunities to build and improve their sense of mutual understanding and solidarity and to facilitate North Korean adolescents' integration into their local communities.

As a result of the intervention, the experimental group's mean psychosocial adaptation score decreased from 75.9 points at the baseline (pretest score) to 73.6 points 1 week after the intervention (posttest score). However, the degree of change was not significantly higher than that of the control group. This was similar to a previous study [3] that found that post-intervention changes in self-esteem and the defense mechanisms of denial, rationalization, and regression were insignificant even after participation in a social integration adaptation program. In a study of children and adolescents from multicultural families, elementary school students from multicultural families had higher scores for depression, anxiety, and hyperactivity than secondary school students from monocultural families; secondary school students from multicultural families also had higher scores for depression, anxiety, and withdrawal [24]. Attention has also been given to the importance of solving problems associated with children from multicultural families to prevent them from developing social problems such as being truant in school, becoming runaways, and developing violent behaviors [24]. In the future, the proposed program should be improved through a series of follow-up studies and by expanding its contents to address participant characteristics other than those covered in this study (withdrawal, anxiety, delinquency, and aggression), such as self-esteem, anger control, and peer attachment [25]. For variables such as depression, self-regulation, self-efficacy, and self-esteem, the significance of research outcomes differed between programs in some cases [26]. Although there was a decrease in the mean score for psychosocial adaptation in this study, it was not statistically significant, likely due to insufficient verification of the adequacy of the program's contents, method of operation, and environment. It is also likely that differences in professionalism between the program facili- tators affected the program's outcomes. In future expansions of the program's content and design, or when local communities create new adaptation programs for North Korean defectors, other psychosocial situational variables-besides the ones mentioned in this study, such as withdrawal, anxiety, delinquency, and aggression-should also be addressed, including self-esteem, anger control, and peer attachment [25]. The subjects of this study were also from various age groups; therefore, future programs should be suited to the level of the participants. There is also a need for a qualitative study, as well as a study design that considers the specificity and continuity of the program, that includes long-term observation. In addition, only highly experienced program organizers should be selected to work with North Korean adolescent defectors.

After the intervention, the mean PTSS score for the experimental group increased slightly from 12.3 points at baseline to 12.9 points, albeit without statistical significance, while the mean PTSS score decreased from 14.5 to 13.6 points in the control group. The degree of change was not statistically significant in either group. This result also highlights the need for regular observation and intervention programs to manage North Korean adolescent defectors' PTSS, especially anxiety, which persists over long periods of time. The continued presence of PTSS may also be due to North Korean adolescent defectors' anxieties about their new environments and uncertain futures, even after they have entered South Korea [17]. This suggests that their PTSS cannot be resolved with short-term educational interventions alone and that management of PTSS among North Korean defectors requires long-term measures that should be provided until they are successfully integrated into their local communities. In addition, a theoretical framework for such intervention programs must be established in order to address the lack of generalizability of the programs developed and implemented so far, given that programs used in previous studies were neither based on a theoretical framework nor validated by intervention studies. 
The experimental group's mean cultural adaptation stress score decreased from 22.9 to 20.4 points, though it was not statistically significant, which is inconsistent with a previous study that found that the mean cultural adaptation stress score of children from multicultural families significantly decreased after a therapeutic singing intervention [18]. It might be difficult for programs to result in significant effects when they are ultimately based on South Korean cultural customs and practices and the North Korean adolescent defectors have not yet developed a strong cultural understanding of South Korea. As a result, North Korean adolescents may actually experience a greater disconnect with South Korean culture after such programs. Cultural adaptation stress experienced by North Korean adolescent defectors was reported to have a strong negative effect on their adaptation to South Korean society [5]. The Rainbow Youth Center run by the Migrant Youth Foundation, a non-profit corporation established in 2006 by the Ministry of Gender Equality and Family, provides adaptation and psychological support services for North Korean adolescent defectors, from admission to Hanawon (the South Korean settlement support center for the re-education of North Korean refugees) to visiting education, after-school education, and comparative cultural experience. However, these services are regionally limited, and a nationwide support network has not yet been established. Negative thoughts and emotions caused by cultural adaptation stress can interfere with the healthy formation of one's self-esteem, which in turn can lead to a lack of self-confidence, passive self-expression, maladaptation to school, difficulty building interpersonal relationships [27], and aggressive behavior [28]. Peer support had a moderating effect on aggressive behavior due to acculturation stress [28]. Therefore, if North Korean adolescent defectors are supported by peers who have an understanding of their characteristics and an active desire for harmonious coexistence, their culture adaptation stress and aggressive behaviors may be alleviated. As such, programs should be supplemented with content designed to improve South Korean adolescents' social awareness, which would result in a decline in prejudice and discrimination, an increase in their respect for cultural differences between them and North Korean adolescent defectors, and a unique understanding of the specific traits of North Korean adolescent defectors. In addition, educational efforts to minimize cultural conflicts at the school and community level could encourage South Korean adolescents to participate in such programs, where they can develop a balanced view of North Korea and learn from the cultural experiences of North Korean defectors to promote mutual understanding [9].

The mean self-efficacy score for the experimental group decreased from 76.2 points after the pretest to 75.9 points after the posttest, while the control group's mean score for self-efficacy increased from 77.9 to 78.6 points, though neither group showed a statistically significant change. This finding is similar to those of studies on integrative social adaptation conducted by $\mathrm{Na}$ [3], which found that self-esteem decreased after the intervention, and Yoon and Kang [18], which found no significant differences in overall self-esteem and schoolrelated self-esteem between the experimental and control groups. The self-efficacy tool is a general scale that did not reflect the situational specificity of North Korean adolescent defectors, and the subjects were believed to have completed the questionnaire without fully understanding it. Self-efficacy develops gradually through repeated experience [7], and a few sessions of a short program may not have been enough to improve the self-efficacy of North Korean adolescent defectors, who tend to experience serious psychological confusion and difficulties. In this study, the subjects' self-efficacy scores were not relatively low in both the experimental and control groups. However, improved self-efficacy, which is an important factor that influences psychosocial adaptation, would be helpful for North Korean adolescent defectors' stable resettlement in South Korea [29]. Self-efficacy is the confidence that one can successfully achieve a specific outcome [7]. If programs are conducted without a clear understanding of their purpose, it may have unintended results and lead to frustration in the participants, which may in turn result in a lower sense of self-efficacy. Therefore, a pre-intervention orientation session should be conducted before the start of such programs in order to carefully confirm whether the participants have thoroughly understood the program's purpose and contents.

The results of this study have limited generalizability to the entire population of North Korean adolescent defectors since a randomized blinded study design could not be used for the small sample size of this study, mainly due to limited accessibility to North Korean adolescent defectors. This problem was aggravated by the fact that some of the participants were Korean-Chinese bilingual students, which partially hindered the flow of the program. The dropout rate was also high. In addition, since it was difficult to compensate for the number of participants who dropped out, the sample size was small in this study, thereby limiting the generalizability of the research findings to all North Korean adolescent defectors. Another limitation of this study concerns procedural problems related to scheduling difficulties, such as conflicts with the start and end times of sessions, absences, and departures, which had an adverse impact on the effectiveness of the program and intervention outcomes.

In follow-up studies, these interventional limitations should be considered at the study design stage. For example, future studies should consider adopting a randomized control trial 
design, adopting a quasi-exponential study design with weekend sessions to avoid scheduling problems, excluding or separating participants with an insufficient command of the Korean language, or expanding the recruitment area and the number of subjects. The validation of the efficacy of the proposed program was limited by the fact that it was designed and implemented without having sufficiently considered the characteristics of North Korean adolescent defectors. This limitation should be addressed in future studies by conducting a preliminary survey on the characteristics of North Korean adolescent defectors using focus group interviews or preintervention interviews with the participants. Programs should be re-examined during development after reviewing their contents and vocabulary, their appropriateness in terms of operating time, and their methods, with the aim of better addressing the needs of North Korean adolescent defectors. In other words, it is necessary to examine whether there are gaps with peers preventing some participants from fully understanding the educational contents of the program and achieving the program's purpose. In addition, intensive camp-type programs should be considered since they encourage participation, prevent a high dropout rate, and can increase participants' interest and motivation. Since adolescence is a period of growth as a result of relationships with peers, interaction with others through camp activities would have a positive effect on the overall development and psychosocial adaptation of the subjects [30]. There is also a need to segment the target audience. In this study, the ages of the recruited subjects ranged from 12 to 20 years, and adolescents from upper elementary school and high school participated in the same program. Since there may be differences in the rate at which students of different ages learn and in the effect of the program on upper elementary and high school students, a segmented program that classifies participants according to age should be devised and implemented to analyze its effects in more depth.

In the future, if the program from this study is modified and implemented based on our results, it is expected to be helpful for the integrated psychosocial adaptation of North Korean adolescent defectors.

\section{CONCLUSION}

This study was conducted in an attempt to develop and administer a program to promote North Korean adolescent defectors' psychosocial adaptation and to examine such a program's effectiveness. In doing so, we aimed to establish a social support network of adolescent peers to provide North and South Korean adolescents with opportunities to build and improve mutual understanding and solidarity and to facilitate the former's integration into their local communities.
No significant differences were observed in the psychometric test scores for psychosocial adaptation, stress, and self-efficacy of North Korean adolescent defectors before and after the program. This finding suggests that psychosocial adaptation, stress, and self-efficacy cannot be improved by short-term interventions. It was also found that the North Korean adolescent defectors' cultural traits had not been sufficiently considered before conducting the program and that difficulties accessing the target population were not accounted for when designing the program's methods. Therefore, in the future, it is essential to provide a sufficient orientation to the participants prior to conducting the program and to include procedures to ensure that they have fully understood the contents and methods of the program. The results of this study also highlight the necessity of regular, active adaptation management for North Korean adolescent defectors even after their successful integration into local communities via long-term research and educational interventions examining their adaptation to South Korean society.

\section{ORCID}

In-Sook Lee $\quad$ https://orcid.org/0000-0003-4156-9685

Jung-Hee Jeon ～https://orcid.org/0000-0002-8045-7064

\section{Authors' contribution}

Conceptualization: all authors; Data collection: all authors; Formal analysis: In-Sook Lee; Writing-original draft: all authors; Writing-review and editing: In-Sook Lee; Final approval of published version: all authors.

\section{Conflict of interest}

No existing or potential conflict of interest relevant to this article was reported.

\section{Funding}

This study was supported by the National Research Foundation of Korea (NRF) grant funded by the South Korean government (No. NRF-2016R1D1A2B03930448).

\section{Data availability}

Please contact the corresponding author for data availability.

\section{Acknowledgements}

None. 


\section{REFERENCES}

1. North Korean Defector Youth Education Support Center. Education for North Korean defectors in 2019 [Internet]. Jincheon: North North Korean Defector Youth Education Support Center; 2019 [cited 2021 March 9]. Available from:

https://www.hub4u.or.kr/hub/edu/status01.do

2. Jeon JY. Mental health problems of adolescents from North Korea: Evaluation and treatment. 2011 Spring conference of the Korean Academy of Pediatric Psychiatry; 2011 April 29; Samsung Medical Center. Seoul: Korean Academy of Child and Adolescent Psychiatry; 2011. p. 140-143.

3. Na YS. A study on social adaptation integrated program for North Korean defectors' children (youth). Korean Journal of 21 Century Social Welfare. 2011;8(1):5-30.

4. Kil EB, Moon SH. The policy implications and problems on social adjustment of North Korean youths in South Korea. Korean Journal of Youth Studies. 2003;10(4):163-186.

5. Kim J, Cho A. The effects on social support and acculturative stress among migrant North Korean adolescents. Journal of Future Oriented Youth Society. 2008;5(2):103-119.

6. Cho YA. The effect of perceived discrimination on psychological distress among North Korean refugees. Korean Journal of Counseling. 2011;12(1):1-19.

https://doi.org/10.15703/kjc.12.1.201103.1

7. Bandura A, Schunk DH. Cultivating competence, self-efficacy, and intrinsic interest through proximal self-motivation. Journal of Personality and Social Psychology. 1981;41(3):586-598. https:// doi.org/10.1037/0022-3514.41.3.586

8. Allen JP, Leadbeater BJ, Aber JL. The relationship of adolescents' expectations and values to delinquency, hard drug use, and unprotected sexual intercourse. Development and Psychopathology. 1990;2(1):85-98.

9. Lee I. Structural equation model for psychosocial adjustment in North Korean adolescent refugees. Child Health Nursing Research. 2018;24(3):287-297. https://doi.org/10.4094/chnr.2018.24.3.287

10. Iizuka CA, Barrett PM, Gillies R, Cook CR, Miller D. The FRIENDS emotional health program for minority groups at risk. Journal of School Health. 2014;84(2):124-132.

https://doi.org/10.1111/josh.12127

11. Sullivan AL, Simonson GR. A systematic review of school-based social-emotional interventions for refugee and war-traumatized youth. Review of Educational Research. 2016;86(2):503-530. https://doi.org/10.3102/0034654315609419

12. Heo EY, Kang H. Development of a Career Guidance Program for the newly settled youths. Journal of Career Education Research. 2010;23(4):39-55.

13. Byun SW, Kim SH, Park SH. A meta-analysis on the effectiveness of interventions applied to teens in multicultural families. Journal of Digital Convergence. 2017;15(1):277-285.

https://doi.org/10.14400/JDC.2017.15.1.277

14. Rousseau C, Drapeau A, Lacroix L, Bagilishya D, Heusch N. Evaluation of a classroom program of creative expression workshops for refugee and immigrant children. Journal of Child Psychology Psychiatry. 2005;46(2):180-185. https://doi.org/10.1111/j.1469-7610.2004.00344.x

15. Fox PG, Rossetti J, Burns KR, Popovich J. Southeast Asian refugee children: A school-based mental health intervention. International Journal of Psychiatric Nursing Research. 2005;11(1):1227-1236.

16. Alayarian A. Trauma, resilience and creativity: Examining our therapeutic approach in working with refugees. European Journal of Psychotherapy and Counselling. 2007;9(3):313-324. https://doi.org/10.1080/13642530701571682

17. Kim HS. The effects of motivation regulation training program on motivation regulation and self-regulated learning of North Korean adolescent refugees in South Korea [master's thesis]. Seoul: Seoul Women's University; 2009. p. 1-125.

18. Yoon SY, Kang KS. The effects of song psychotherapy on the acculturation stress and self-esteem of children from multicultural families: pilot study. Korean Journal of Music Therapy. 2016;18(2): 119-138. https://doi.org/10.21330/kjmt.2016.18.2.119

19. Oh KJ, Lee HR, Hong KE, Ha EH. K-YSR: Korean youth self report. Seoul: Chung Ang Aptitude Publishing Co.; 1997.

20. Achenbach TM. Manual for the youth self-report and 1991 profile. Burlington, VT: University of Vermont Department of Psychiatry; 1991.

21. Kim YH, Kim HA, Cho YA, Kim YJ, Lee GR. A study on development of psychological and social adaptation evaluation tool for North Korean deviant youth. Seoul: Rainbow Youth Center; 2009.

22. Chung KH. A study on the self-efficacy effect of North Korean escapee students using drum [master's thesis]. Seoul: Chung-Ang University; 2017. p. 1-88.

23. Cha JE. A study for development for a general self-efficacy scale. Psychological Research. 1996;34:19-31.

24. Jang DH, Shin HS. The environmental factors on the school maladjustment of children from multicultural families. Korean Journal of Youth Studies. 2010;17(3):123-147.

25. Miller LD, Gold S, Laye-Gindhu A, Martinez YJ, Yu CM, Waechtler V. Transporting a school-based intervention for social anxiety in Canadian adolescents. Canadian Journal of Behavioural Science. 2011;43(4): 287-296. https:// doi.org/10.1037/a0023174

26. Kim YR, Yoo SH, An J. Research trends in group programs for institutionalized children and adolescents. Studies on Korean Youth. 2018;29(3):71-99. https://doi.org/10.14816/sky.2018.29.3.71

27. Kim EG, Kim JN. The relationships between multi-cultural adolescents' acculturation stress and psycho-social adjustment: The mediating effect of ego-identity and the moderating effect of perceived social support. Multicultural Education Studies. 2016;9(4): 21-43. https://doi.org/10.14328/MES.2016.12.31.21 
28. Ah YA. The effects of acculturation stress on aggressive behavior in North Korean adolescent defectors and the regulating effects of social support. Korean Journal of Youth Studies. 2019;26(5):159-182. https://doi.org/10.21509/KJYS.2019.05.26.5.159

29. Ko KS, Kim HT. Factors influencing the psycho-social adaptation of koryuin who reside in Korea. Journal of Church Social Work. 2011;15:45-84.

30. Sibthorp J, Wilson C, Povilaitis V, Browne L. Active ingredients of learning at summer camp. Journal of Outdoor and Environmental Education. 2020;23:21-37. 